\title{
Metagrating solutions for full color single-plate waveguide combiner
}

\author{
Oksana Shramkova*, Valter Drazic, Guillaume Bourcin, Bobin Varghese, Laurent Blondé, and Valérie Allié \\ InterDigital R\&D France, Immersive Media Lab., 975 Avenue des Champs Blancs, 35576 Cesson Sévigné, France
}

Received: 15 October 2021 / Accepted: 17 January 2022

\begin{abstract}
In this work we propose several full-color metagrating solutions for single waveguide-based Augmented and Virtual Reality near-eye display systems. The presented solutions are based on a combination of reflective and/or transmissive diffraction gratings inside or outside a waveguide. The proposed in-coupler designs have high diffraction efficiency across a wide angular range. Applying our new grating combination solution, we can provide good gathering of diffracted rays for the different colors. We demonstrate that by using a dual-mode symmetrical in-coupling system and angular pupil tiling, we can extend the overall horizontal FoV for three RGB colors. The new characteristics of the full single waveguide system including Eye Pupil Expander and out-coupling components compatible with the proposed in-coupling solutions are discussed. We show that a new nonsymmetrical design of metagratings can be used to change its diffraction properties improving the diffraction efficiency and diffraction uniformity of the optical components.
\end{abstract}

Keywords: Optical combiner for AR glasses / diffractive gratings / metasurface / edge waves

\section{Introduction}

Augmented/virtual reality $(\mathrm{AR} / \mathrm{VR})$ is a fast-growing technology trend that has a broad range of applications. Optical see-through head mounted display is a key device for $\mathrm{AR} / \mathrm{VR}$ applications attracting significant interest of major industrial players $[1-3]$. To realize a compact neareye display system, various technologies have been developed [4-11]. Currently most of the head mounted displays use the waveguide structure in order to reduce the overall size and weight of the device [1]. The basic optical components integrated into the waveguide combiners consist of in- and out-couplers, and the Eye Pupil Expander (EPE) which are fabricated as diffractive optical elements [12,13] or holographic volume gratings [14,15]. Some of kinds of AR/VR glasses utilize optical waveguides wherein light propagates into the optical waveguide by Total Internal Reflection (TIR). The Field of View (FoV) of the waveguide depends on the waveguide material refractive index. To couple light into the waveguide and provide good color uniformity, the main diffracted order should have high diffraction efficiency across a wide angular range. Such functionality can be achieved by using single-mode nonsymmetrical gratings $[1,12]$ with nonsymmetrical

\footnotetext{
* e-mail: Oksana.Shramkova@interdigital.com;

o.shramkova@gmail.com
}

optical power distribution between diffraction orders ( $T_{j} \neq T_{-j}, R_{j} \neq R_{-j} \ldots$., where $j$ is the number of diffraction order, $T$ and $R$ are the transmissivity and reflectivity of the gratings) leading to maximal grating efficiency for desired diffraction order. Changing the period of nonsymmetrical gratings we can get on-axis (symmetrical) or offaxis (asymmetrical) in-coupling for the desired FoV of the device. To develop a waveguide display system with a wide FoV we can tile angularly the exit pupil of the lightengine. In such a system the angular exit pupil of the system is split by the grating in such a way that one portion is diffracted into a first direction into the waveguide, and the remaining portion into the opposite direction. Usually, the exit pupil is split with respect to the waveguide's normal, it means that the rays corresponding to positive and negative angles of an incidence will be diffracted by the system into opposite directions. Such a system doubles the FoV as each half-image can use the whole angular bandwidth of the waveguide in each direction of propagation. To provide such functionality, we can use a dual-mode system with symmetrical gratings $[13,16]$ which achieves symmetrical response between the orders $\left(T_{j}=T_{-\mathrm{j}}, R_{j}=R_{-j}\right)$, or combine two single-mode nonsymmetrical gratings $[12,17-19]$. In the last systems to get similar functionality, the rays corresponding to positive and negative angles will be diffracted by two differently oriented nonsymmetrical gratings. The exit pupil will be reconstructed again at the eyebox by a 


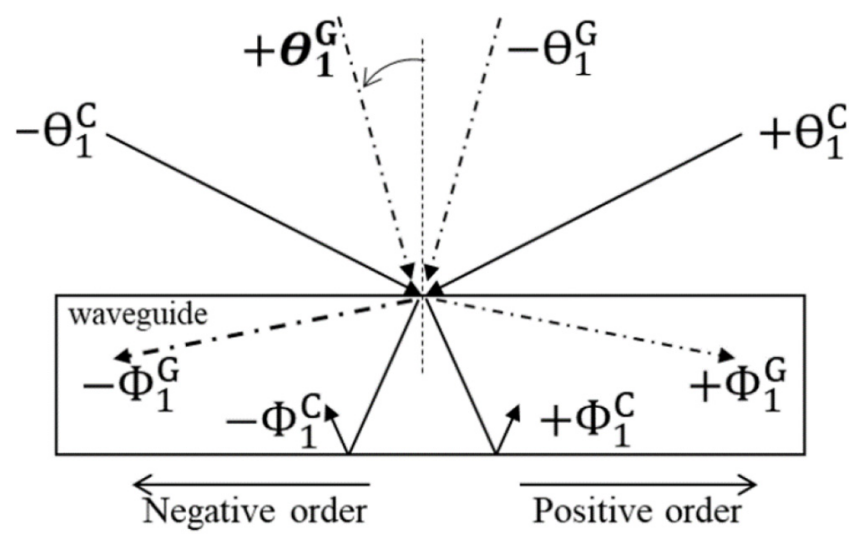

Fig. 1. Schematic of dual-mode diffraction grating design with overlap of incident rays.

properly oriented out-coupler, and the users will see one single virtual image.

In Figure 1 we have illustrated one important way of using symmetric diffraction gratings to achieve pupil tilling and to double the FoV of a single wavelength system by using two propagation directions inside of a flat waveguide. The period of the transmissive diffraction grating was selected for a particular wavelength and for an angular range covering overall $\mathrm{FoV}$, assuming that the rays incident on the in-coupler with angles $\pm \theta_{1}^{C}$ get diffracted inside the waveguide with critical angles $\pm \Phi_{1}^{C}$, while those with angles $\pm \theta_{1}^{G}$ in the vicinity of the normal get diffracted with grazing angles $\pm \Phi_{1}^{C}$, respectively (see Fig. 1). This means that all rays on the right-hand side of $+\theta_{1}^{G}$ get diffracted into the waveguide and propagate to the righthand side and those on the left-hand side of $-\theta_{1}^{G}$ get diffracted into the waveguide and propagate toward the left-hand side. We fix $\Phi_{1}^{G}=75^{\circ}$.

One of the most serious challenges in $\mathrm{AR} / \mathrm{VR}$ is a fullcolor solution with a large overall FoV. Several full-color waveguide solutions have been developed for light in-coupling into the optical device [1]. In order to provide the transmission of multiple RGB wavelengths with a large FoV, we can fabricate a multi-waveguide solution combining stacked waveguides, each optimized for a different color. Reducing the number of waveguides while keeping the largest possible FoV allowed by the parameters of the waveguide material is the key challenge since it miniaturizes and simplifies the system. For creating a waveguide display system, we must take into account that the EPE component sets a limitation on the vertical FoV [20]. The main role of EPE component is to deviate a wide optical beam and duplicate a single pupil into many. The EPE gratings are TIR diffraction gratings in conical mounting [19,21]. As it will be demonstrated below, the design of the EPE component requires a careful calculation of the grating's pitch in order to optimize it for the best possible vertical FoV.

Our single-waveguide full-color solutions for symmetrical and nonsymmetrical in-couplers providing high in-coupled efficiency across a wide angular range are presented in Section 2. The proposed designs are based on metagrating solutions that operate to combine the beams diffracted by combinations of the reflective and/or transmissive gratings. Considered metagratings consist of a periodic arrangement of high refractive index sub-wavelength elements. In Section 3 we show a new EPE component which can be compatible with the full-color in-coupler. The main findings are summarized in the conclusion section.

\section{Overview of the in-coupling structures}

In this section we describe single waveguide dual- and single-mode metagrating systems for in-coupling light into an optical device. Constitutive parts of proposed metagrating solutions are different diffraction gratings having different periods calculated for the proper wavelength and may have different size and material of the elements. The geometrical structures of the elements are configured to emphasize edge-waves (EWs) [13] diffracted by the vertical edges of the high-refractive index elements. Schematic cross-sectional views of a waveguide with 3 types of metagrating in-couplers inside and outside the waveguide are presented in Figure 2.

\subsection{Dual-mode metagrating solution}

First proposed design (see Fig. 2a) is based on a dual-mode metagrating solution inside a waveguide that operates to combine the beams diffracted by the reflective grating on the top of metagrating system and transmissive diffraction grating at the bottom of the system. To calculate the periods of both gratings, we assume that the largest angular span that can be coupled propagates into the waveguide between TIR and a grazing angular limit.

In Figure 3 we have schematically depicted the angular distribution of the incident and diffracted angles for the three colors. The pitch size of the reflective diffraction grating $d_{1}$ should be selected for blue color wavelength and an angular range covering full $\mathrm{FoV}$ allowed by the parameters of the waveguide. We assume that the rays incident on the in-coupler with angles $\pm \theta_{1}^{C}$ get diffracted inside the waveguide with critical angles $\pm \Phi_{1}^{C}$, while those with angles $\pm \theta_{1}^{G}$ in the vicinity of the normal get diffracted with grazing angles $\pm \Phi_{1}^{G}$, respectively (see Fig. 3a). We fix $\Phi_{1}^{G}=75^{\circ}$. The total allowed horizontal FoV for the symmetrical, dual-mode system could be determined as:

$$
\Delta Q^{H}=2 \sin ^{-1}\left(\frac{n_{2}-1}{2}\right),
$$

where $n_{2}$ is the refractive index of the waveguide. For the wavelengths corresponding to the green and red colors we observe a shift of in-coupled angular distribution toward the higher angles of incidence (see Fig. 3b,c). For both colors, the angular range $\left[-\theta_{1}^{G} ; \theta_{1}^{G}\right]$ transmits through the first reflective diffraction grating with a very high efficiency for 0th transmitted order. This portion of incident image will be diffracted by the second transmissive grating which is on the bottom of metagrating composition. The second diffraction grating is different from the first one in that it has a different pitch size, but the geometrical structure can be of the same shape. The pitch size $d_{2}$ of reflective diffraction grating should be calculated for the red color wavelength and an angular range covering full FoV for the 


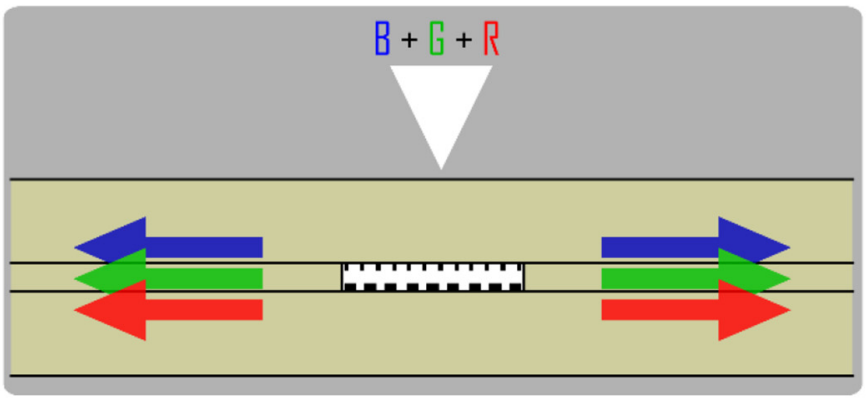

(a)

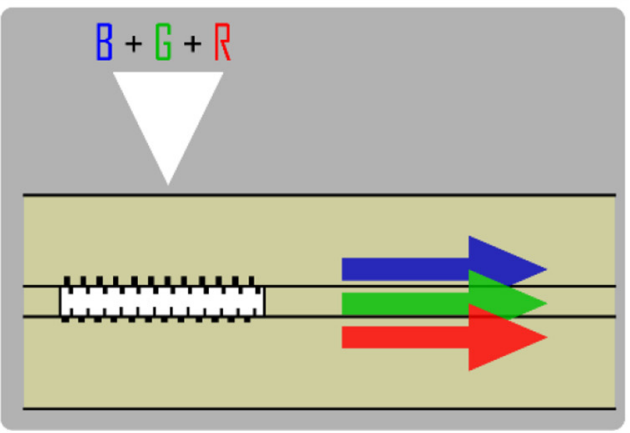

(b)

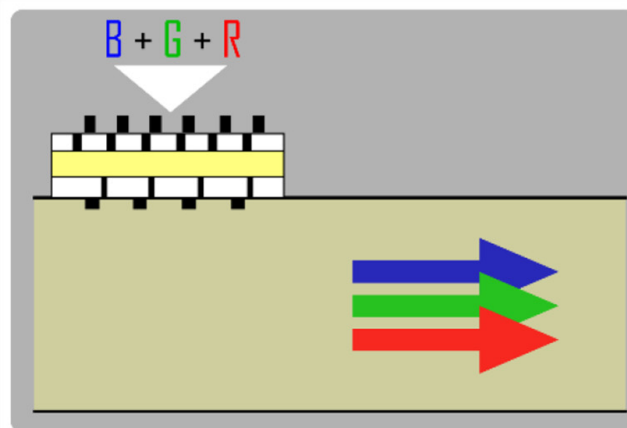

(c)

Fig. 2. Schematic side view of the single-waveguide full color system for light in-coupling into the optical device: (a) dual-mode embedded metagrating solution; (b) single-mode embedded metagrating solution; (c) single-mode transmissive metasurface.

transmissive diffraction grating, also assuming that the rays diffracting into the grazing direction into the waveguide (the grazing rays) are hitting the grating in the vicinity of the normal. This diffraction grating should be also optimized to get high diffraction efficiency of in-coupled orders in the mentioned angular range at the red color wavelength. At the wavelength corresponding to the green color, we will observe a shift of an angular distribution toward the lower angles of incidence leading to the angular overlapping of corresponding positive/ negative diffraction orders.

Using the system of diffraction grating equation we get the equations for the periods of reflective and transmissive diffraction gratings which can be presented in such form:

$$
\begin{aligned}
& d_{1}=M_{R} \frac{\lambda_{\text {blue }}}{n_{2} \sin \Phi_{1}^{G}-n_{1} \sin \theta_{1}^{G}}, \\
& d_{2}=M_{T} \frac{\lambda_{\text {red }}}{n_{2} \sin \Phi_{2}^{G}+n_{1} \sin \theta_{2}^{G}} .
\end{aligned}
$$

Here $n_{1}$ is the index of the surrounding media.

The general topology of the proposed metagrating unit cell is illustrated in Figure 4a. To estimate the effectiveness of the full system, we consider the unit cell comprising four elements with refractive index $n_{4}$ and three elements with refractive index $n_{3}$. As a result, the period of the metagrating is equal to $d=4 d_{1}=3 d_{2}$. If the period of reflective diffraction grating is defined to in-couple diffraction order $M_{\mathrm{R}}$ and the period of transmissive diffraction grating is defined to in-couple diffraction order $M_{\mathrm{T}}$, then the period of the new metagrating is defined to in-couple reflected order $M_{\mathrm{R}}{ }^{*}=4 M_{\mathrm{R}}$ and transmitted order $M_{\mathrm{T}}{ }^{*}=3 M_{\mathrm{T}}$. The cross-sectional view of metagrating unit cell corresponds to high refractive index $\left(n_{4}\right)$ elements on the bottom of a homogeneous dielectric plate with refractive index $n_{2}\left(n_{4}>n_{2}\right)$. We define $d_{1}$ as the period of this first grating, $w_{1}$ and $h_{1}$ are the width and height of the high refractive index elements. The second part of the proposed metagrating contains high refractive index $\left(n_{3}\right)$ elements on top of a homogeneous dielectric plate with a refractive index $n_{2}\left(n_{3}>n_{2}\right)$. We assume that $d_{2}$ is the period of the second grating, $w_{2}$ and $h_{2}$ are the width and height of the high refractive index element. To get the unit cell of diffraction metagrating we combine two plates with first (DG1) and second (DG2) diffraction gratings. The distance between the substrate surfaces is equal to $h_{1}+h_{2}+h_{\mathrm{a}}$, where $h_{\mathrm{a}}$ is the vertical distance between the elements of DG1 and DG2. The volume between the elements of the two gratings and substrate surfaces may be empty or may be filled by a material with low refractive index $n_{1}\left(n_{1}<n_{2}\right)$. Here, we assume this material is air. The materials and size of the constitutive parts of the diffraction grating elements can be optimized to manage the position, direction, phase, and amplitude of the EWs diffracted by the elements [13].

A linearly polarized plane wave is incident on the metagrating system from the top in a plane perpendicular to the metagrating. We must note that the proposed 


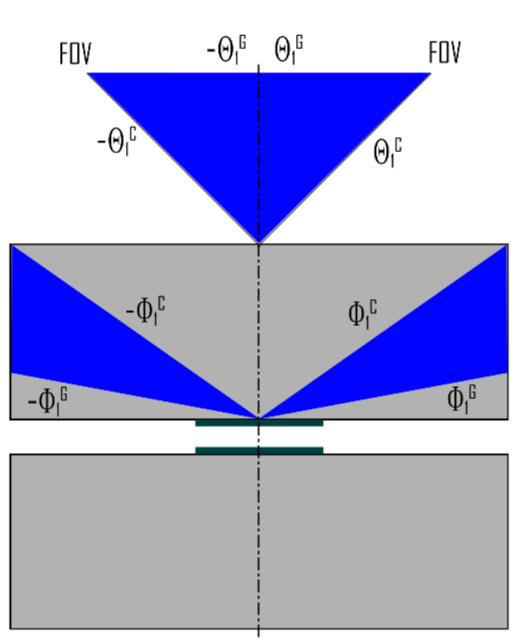

(a)

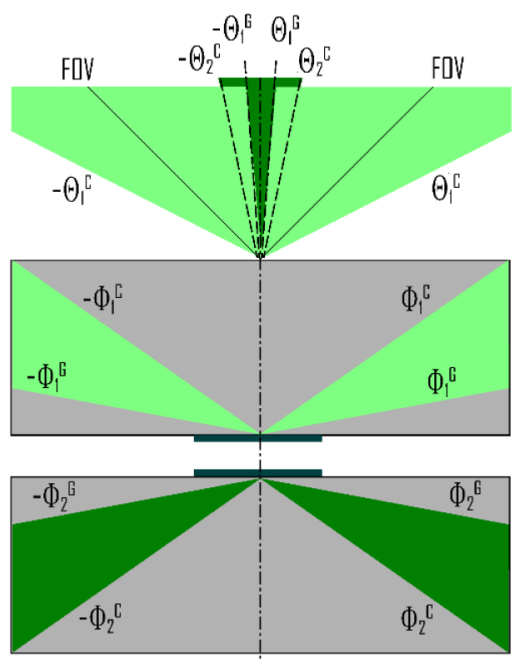

(b)

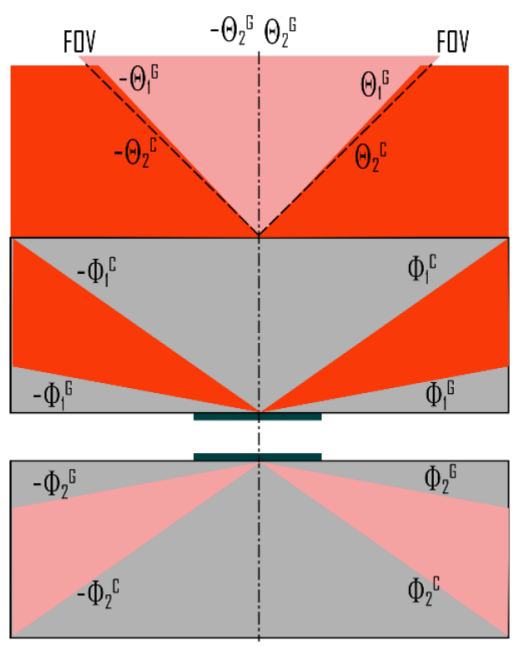

(c)

Fig. 3. Schematics of a single waveguide system with dual-mode embedded metagrating solution illustrating angles of incident and diffracted light for reflective and transmissive diffraction gratings for three different colors: (a) blue, (b) green, (c) red. Angles denoted using $\theta$ are located in the air. Angles denoted using $\Phi$ are located in the waveguides and measure the angle of rays that have been diffracted. Superscript C indicates a critical ray (just at the TIR limit in the waveguide), either in air or in the waveguide. Superscript G indicates a grazing ray (the highest allowed angle of incidence in the waveguide).

Incident wave

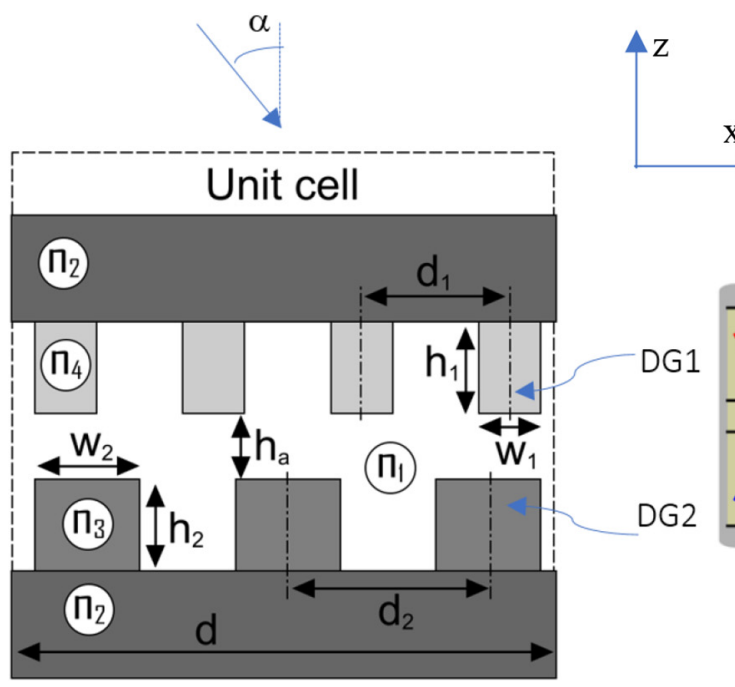

(a)

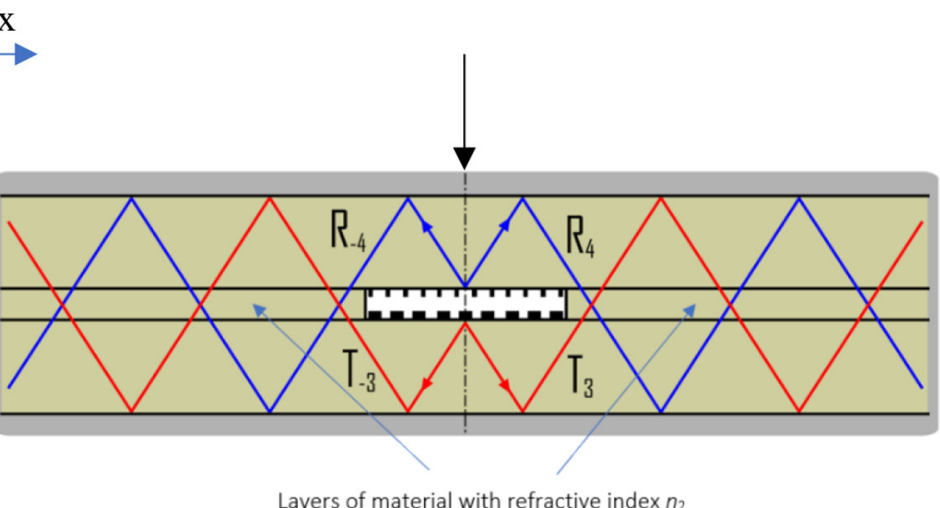

(b)

Fig. 4. (a) Cross-sectional view of the unit cell of metagrating; (b) geometry and distribution of diffraction order propagation. In this example use of a metagrating, the fourth reflected order $\mathrm{R}_{ \pm 4}\left(M_{\mathrm{R}}{ }^{*}=4\right)$ and third transmitted order $\mathrm{T}_{ \pm 3}\left(M_{\mathrm{T}}{ }^{*}=3\right)$ will be in-coupled into the waveguide.

solution applies for TE and TM polarizations. However, to get the maximal efficiency, the system should be optimized considering the polarization of the incident wave.

The proposed topology applies to a dual symmetrical diffraction grating system where the pupil is split angularly. To provide total reflection of the diffracted light only by the external (horizontal) walls of the waveguide, a thin layer of thickness $h_{1}+h_{2}+h_{\mathrm{a}}$ and with refractive index $n_{2}$ may be positioned between the plates with the diffraction gratings.

Selecting the reflective (DG1) and transmissive parts (DG2) of the metagrating to in-couple first diffraction orders $\left(M_{\mathrm{R}}=M_{\mathrm{T}}=1\right)$ results in a metagrating in which the fourth reflected order $\mathrm{R}_{ \pm 4}\left(M_{\mathrm{R}}{ }^{*}=4\right)$ and the third 


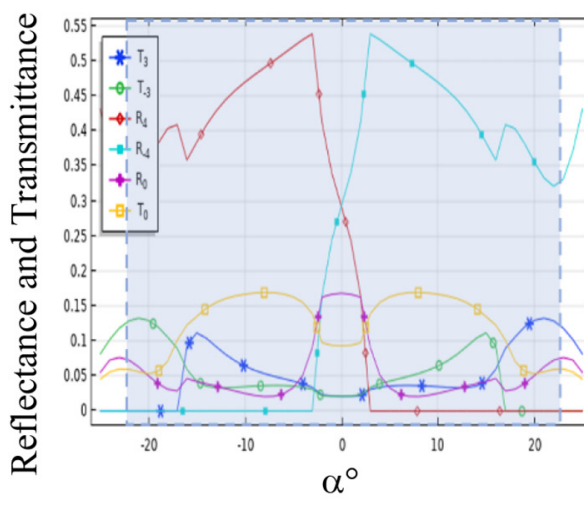

(a)

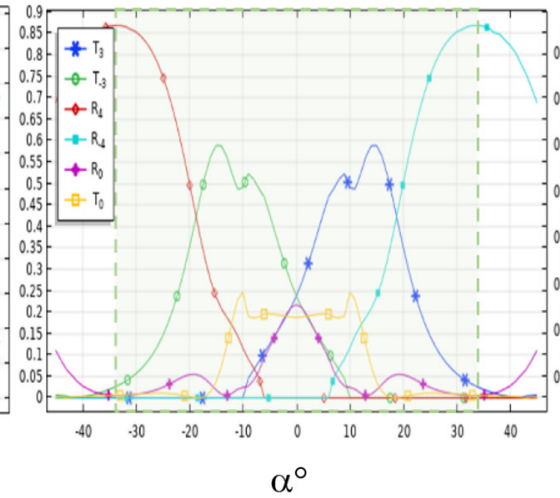

(b)

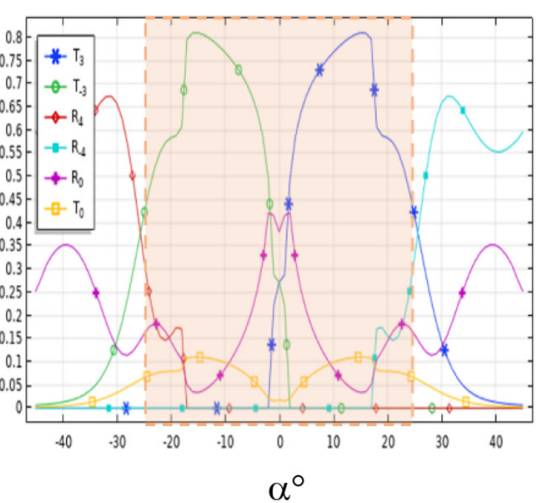

(c)

Fig. 5. Diffraction performance of the AlAs/Si metagrating for (a) blue, (b) green and (c) red light. The angular ranges in-coupled by the system are shaded. The angular ranges correspond to an incidence from the medium with refractive index $n_{2}$.

transmitted order $\mathrm{T}_{ \pm 3}\left(M_{\mathrm{T}}{ }^{*}=3\right)$ will be in-coupled into the waveguide (see Fig. 4b). To prevent undesirable diffraction of reflected $\mathrm{R}_{ \pm 4}$ and transmitted $\mathrm{T}_{ \pm 3}$ orders, the lateral size of the metagrating may be considered in the selection of the width of the plates as well as the thicknesses of the first and second plate, with reflection and transmission gratings.

Assuming that the refractive index of the waveguide material is $n_{2} \approx 1.76$ and some angles are overlapped near normal incidence in symmetric diffraction directions, to avoid missing display information for some colors [13], we obtain an overall horizontal $\mathrm{FoV}$ of $89^{\circ}$ for the proposed solution.

Figure 4 illustrates diffraction performance of a metagrating using the unit cell with the following parameters: $w_{1}=100 \mathrm{~nm}, \quad h_{1}=240 \mathrm{~nm}, \quad w_{2}=80 \mathrm{~nm}$; $h_{2}=100 \mathrm{~nm}, \quad h_{\mathrm{a}}=250 \mathrm{~nm}$. They show the computed transmittance and reflectance of TE-polarized incident wave for blue $\left(\lambda_{\text {blue }}=460 \mathrm{~nm}\right)$, green $\left(\lambda_{\text {green }}=530 \mathrm{~nm}\right)$ and red light $\left(\lambda_{\text {red }}=620 \mathrm{~nm}\right)$ by the metagrating with the period $d=1082.2 \mathrm{~nm}$ and $n_{2} \approx 1.76$. For the system design, Aluminum arsenide (AlAs) was used as material for the elements of the reflective part (elements with refractive index $n_{4}$ ), Silicon ( $\mathrm{Si}$ ) was the material for the elements for the transmissive part (elements with refractive index $n_{3}$ ). The distance between the elements of gratings was filled by air. To increase diffraction uniformity for the light transmitted through the metagrating, the system uses a phase-modifying layer and a stop layer below the elements of second diffraction grating [13]. The high refractive index phase modifying layer is placed on the top of substrate with refractive index $n_{2}$. To simplify the fabrication process and control the depth in the etching process, a stop layer is placed between the elements with high refractive index and phase-modifying layer. For the simulations presented in Figure $5, \mathrm{TiO}_{2}$ has been used as the material of phasemodifying layer and $\mathrm{Al}_{2} \mathrm{O}_{3}$ has been used as the stop-layer material. Thickness of additional layers was $10 \mathrm{~nm}$. The presented data were obtained using COMSOL Multiphysics software. Full-wave electromagnetic analysis was done for an infinite array of the unit cells presented in Figure 4a. We assumed that the system is also infinite in the Y-direction. The angular range presented in Figure 5 corresponds to an incidence from the medium with refractive index $n_{2}$. It can be seen, that the presented metagrating design is configured to in-couple \pm 4 th reflective diffraction orders and \pm 3 rd transmissive diffraction orders for TE polarization. For the proposed solution we can achieve an average diffraction efficiency of $50 \%$ for blue color, of $65 \%$ for green and of $70 \%$ for red over a FoV of $89^{\circ}$. Let us note that the mutual positioning of the elements of 2 diffraction gratings in relation to one another inside the pitch does not affect the system performance.

Let us note that the in-coupling metagrating is transparent for real image and can be also used for outcoupling purposes. The out-coupling grating can be similar to or the same as the in-coupler but reversed top-down to reflect blue and transmit red light. Such an out-coupler will be able also reflect and transmit green. Because the grating is embedded into the waveguide, this metagrating is protected from mechanical damage and degradation.

\subsection{Single-mode metagrating solution}

Very high FoV systems are considered for high end XR and entertainment use cases. For some AR use cases such as smart glasses, targeted FoV is much lower and should lead to less complex system design. Therefore a single-sided diffraction might be sufficient.

In this section we will describe a new embedded RGB single-sided metagrating based on high refractive index material transmissive and reflective diffraction gratings with nonsymmetrical topology. We will show that this topology can increase the diffraction efficiency and diffraction uniformity of the grating. To get on-axis or off-axis in-coupling for the desired FoV of the device, only one single diffraction mode is used to carry the image. Using a waveguide display system with single nonsymmetrical in-coupling grating, we reduce the FoV but simplify the full system configuration.

The general topology of an asymmetrical element of a transmissive diffraction grating unit cell is illustrated in Figure 6 . This cross-sectional view corresponds to the high refractive index $\left(n_{2}\right.$ and $n_{4}$, where $n_{2}$ could be equal to $\left.n_{4}\right)$ 


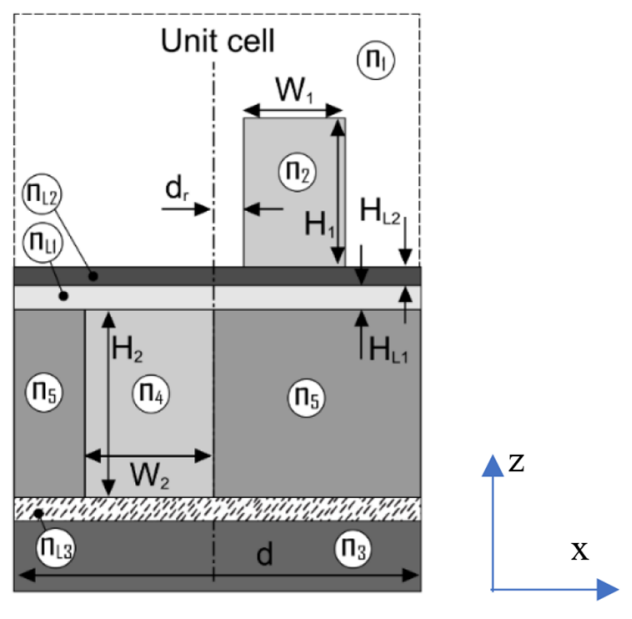

(a)

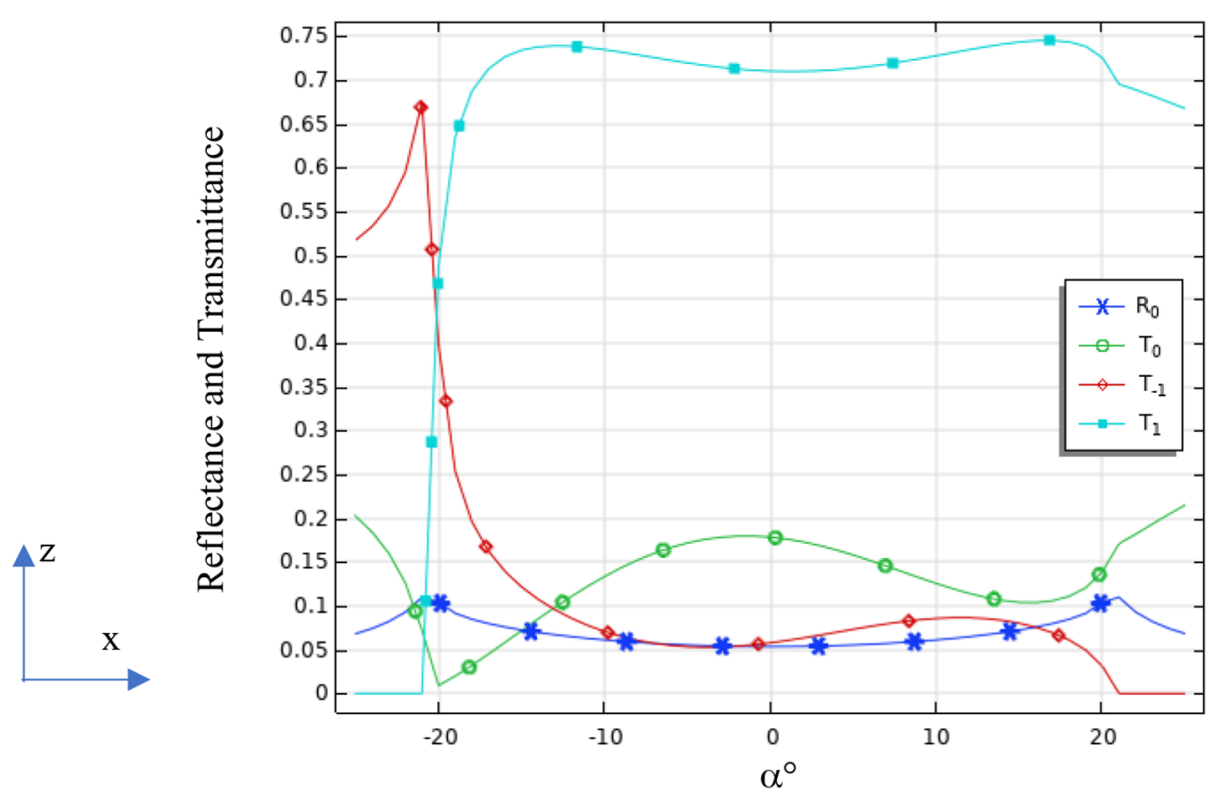

(b)

Fig. 6. (a) Cross-sectional view of transmissive grating unit cell; (b) reflectance and transmittance vs. angle of TE-polarized electromagnetic wave incidence $\alpha$ at $\lambda=625 \mathrm{~nm}, n_{1}=1.0, n_{2}=n_{4}=n_{\mathrm{L} 1}=2.5884, n_{3}=1.7, n_{\mathrm{L} 2}=1.7663, n_{5}=1.4572$ for TiO transmissive $_{2}$ grating with such parameters: $w_{1}=60 \mathrm{~nm}, w_{2}=120 \mathrm{~nm}, h_{1}=170 \mathrm{~nm}, h_{2}=130 \mathrm{~nm}, H_{\mathrm{L} 3}=0 \mathrm{~nm}, d_{\mathrm{r}}=-5 \mathrm{~nm}, H_{\mathrm{L} 1}=H_{\mathrm{L} 2}=5 \mathrm{~nm}$.

elements on the top of homogeneous dielectric plate with a refractive index $n_{3}\left(n_{2,4}>n_{3}\right), w_{1}$ and $h_{1}$ are width and height of the high refractive index elements outside the substrate in host medium with refractive index $n_{1}, w_{2}$ and $h_{2}$ are width and height of the high refractive index elements inside the layer with the refractive index $n_{5}\left(n_{1}<n_{5}<n_{3}\right)$. Parameter $d_{\mathrm{r}}$ describes the mutual position of the top and bottom elements in the grating. It corresponds to the distance between the right vertical edge of the bottom element and left vertical edge of the top element. For negative values of $d_{\mathrm{r}}$, the top element is shifted toward the left with respect to the vertical line corresponding to the position of the right vertical edge of the bottom element. For positive values of $d_{r}$, the top element is shifted toward the right. The period of the diffraction grating is $d$. We assume that a linearly polarized plane wave illuminates the grating from the top in a plane perpendicular to the grating.

To improve the uniformity and additionally increase the transmittivity of the in-coupled diffraction order, we propose to use a phase modifying layer [13] with thickness $H_{\mathrm{L} 1}$ and refractive index $n_{\mathrm{L} 1}$ placed between the high refractive index elements on the top of the layer with refractive index $n_{5}$ and embedded element with refractive index $n_{4}$. The utilization of an additional high refractive index layer between the top and bottom elements of diffraction grating modifies the phase of refracted edge wave providing higher transmissivity of an in-coupled order. To simplify the fabrication process, we can include a stop layer between this thin layer and top element of the grating. $n_{\mathrm{L} 2}$ is the stop layer material refractive index and $H_{\mathrm{L} 2}$ is the thickness of this layer. To control the etching depth of material with refractive index $n_{5}$, we can also use a second stop layer with refractive index $n_{\mathrm{L} 3}\left(n_{\mathrm{L} 3}\right.$ could be equal to $\left.n_{\mathrm{L} 2}\right)$ and the thickness of this layer $H_{\mathrm{L} 3}$.
This nonsymmetrical topology provides the generation of EWs, originating from the edges of the system, contributing to the formation of a final wavefront deflected from the direction of refracted wave [13]. The characteristics of the generated EWs, obtained due to the interference of the EW with the refracted plane wave, are affected by the parameters of the corresponding parts of the nonsymmetrical system, such as refractive index ratios between the dielectric materials forming the system, dimensions of the elements with higher refractive index, and angle of incidence of an incident wave.

Let us consider the performance of a diffraction grating based on the asymmetric high refractive index material elements. As it was mentioned before, the nonsymmetrical diffraction gratings achieve an asymmetrical distribution of diffracted light efficiency. For the simulated case we assume that the first diffraction order is in-coupled into the waveguide. So, it is desirable for the maximal input for a single mode system to correspond to the diffraction order +1 . The computed reflectance and transmittance for TE incidence at $\lambda=625 \mathrm{~nm}$ for a grating with the substrate with $n_{3}=1.7$ and with the period $d=463 \mathrm{~nm}$ is plotted in Figure $6 \mathrm{~b}$. We use $\mathrm{TiO}_{2}$ as the material of the elements of the grating and phase modifying layer and $\mathrm{SiO}_{2}$ as the host medium for the second embedded element of the grating. $\mathrm{Al}_{2} \mathrm{O}_{3}$ is used as the material of the stop layer. Full-wave electromagnetic analysis was done for a one-dimensional periodic array of the elements. We assume that the system is infinite in X-and Y-directions. Such high refractive index material diffraction grating has very high efficiency for transmitted first order. The maximal efficiency for this case is equal to $\eta_{\max }=75 \%$. In order to give a measure for the homogeneity of the diffraction efficiency for all angles of incidence in-coupled into the waveguide, the diffraction 


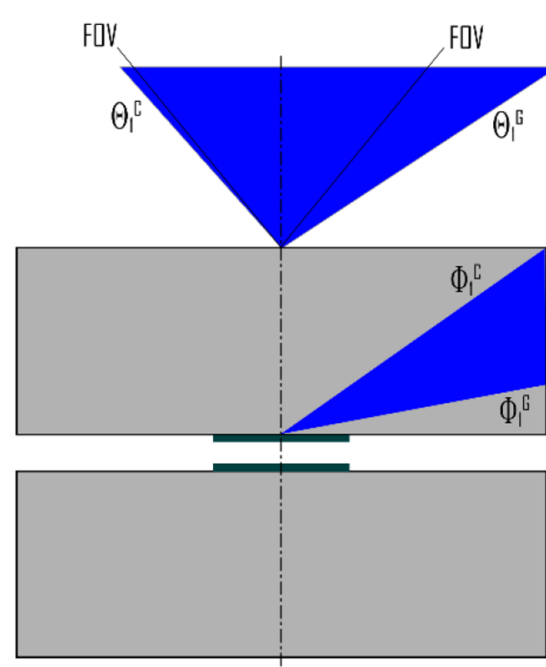

(a)

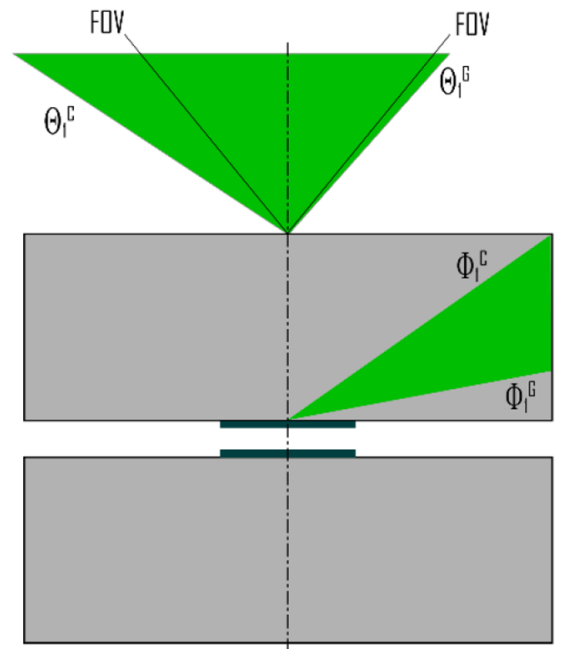

(b)

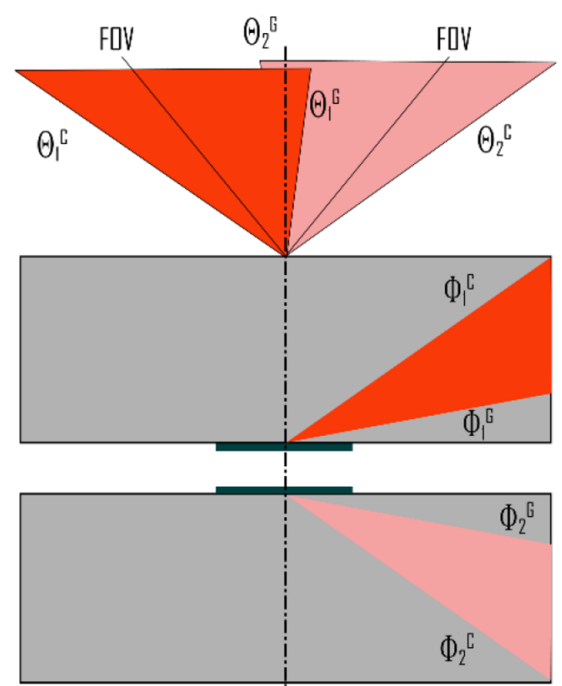

(c)

Fig. 7. Schematic side view of a single waveguide system with symmetrical field of view for three different colors: (a) blue, (b) green, (c) red.

uniformity may be represented as:

$$
G=1-\frac{\eta_{\max }-\eta_{\min }}{\eta_{\max }+\eta_{\min }}
$$

where $\eta_{\max , \min }$ are correspondingly maximal and minimal transmitted efficiency. It was obtained that for considered nonsymmetric high refractive index material diffraction grating over a wide angular range $\left(38^{\circ}\right)$ the diffraction uniformity is about $97.3 \%$.

It was demonstrated that similar topology can be used for fabrication of a reflective diffraction grating with high diffraction uniformity and efficiency for incoupled diffraction orders. To provide a full RGB single waveguide system, we can employ a metagrating inside the waveguide, which will be based on single mode asymmetric reflective and transmissive diffraction gratings described above.

For a new type of metagrating in-coupler the configuration may be performed as follows. For the first reflective diffraction grating DG1 (with the period $d_{1}$, top of metagrating composition), the period of the reflective diffraction grating DG1 may be calculated to in-couple blue and green color wavelengths in the angular range covering the desired $\mathrm{FoV}$ of the device for these two colors, assuming that for blue color the incoming rays with critical angle of incidence are in the vicinity of the lower boundary of the FoV $\left(\theta_{1, b l u e}^{C} \approx \theta_{m}\right)$ and for green color the incoming grazing rays are in the vicinity of upper boundary of the FoV $\left(\theta_{1, \text { green }}^{G} \approx \theta_{M}\right)$, where angles $\theta_{m}$ and $\theta_{M}$ correspond to the lower and upper boundaries of the desired field of view of the proposed device. The diffraction grating may be configured to get high diffraction efficiency of corresponding orders $M_{R}$ in the mentioned angular range at blue and green color wavelengths. From Figure 7a corresponding to the blue color, the angular range $\left[\theta_{1, \text { blue }}^{C} ; \theta_{1, \text { blue }}^{G}\right]$ diffracts inside the waveguide into the angular range $\left[\Phi_{1, b l u e}^{C} ; \Phi_{1, b l u e}^{G}\right]$. Using the system of diffraction grating equations we can select the pitch of first diffraction grating and estimate a minimal refractive index of the waveguide at green color wavelength $\left(n_{3 G}\right)$ in-coupling both colors:

$$
\begin{gathered}
d_{1}=\frac{M_{R} \lambda_{\text {blue }}}{1-n_{1} \sin \left(\theta_{m}+\gamma_{m}\right)}, \\
n_{3 G} \approx \frac{M_{R} \lambda_{\text {green }}}{d_{1}}+n_{1} \sin \theta_{M} .
\end{gathered}
$$

To get this equation we assumed that $\Phi_{1, \text { green }}^{G} \approx 90^{\circ}, \gamma_{m}$ is minimal differences between theoretically possible value of in-coupled incident angle and the required incident angle.

At the wavelength corresponding to green color, we will observe the shift of an angular distribution toward the lower angles of an incidence. As seen in Figure 7b, at green color wavelength the angular range $\left[\theta_{1, \text { green }}^{C} ; \theta_{1, \text { green }}^{G}\right]$ diffracts inside of the waveguide into the angular range $\left[\Phi_{1, \text { green }}^{C} ; \Phi_{1, \text { green }}^{G}\right]$. Similar functionality will be observed at the wavelength corresponding to red color. Increasing the wavelength, we obtain an additional shift of angular distribution toward the lower angles of incidence. As seen in Figure $7 \mathrm{c}$, angles above $\theta_{1 \text {,red }}^{G}$ transmit through the reflective diffraction grating (it corresponds to the 0 transmitted order $\mathrm{T}_{0}$ ) with a high efficiency. This portion of incident image will be also diffracted by transmissive grating DG2, and after it can be combined with the portion of image reflected by the first grating DG1.

The second transmissive diffraction grating DG2 (with the period $d_{2}$, bottom of metagrating composition) may be configured as follows. The period of transmissive diffraction grating DG2 must be calculated for red color 


\section{Unit cell}
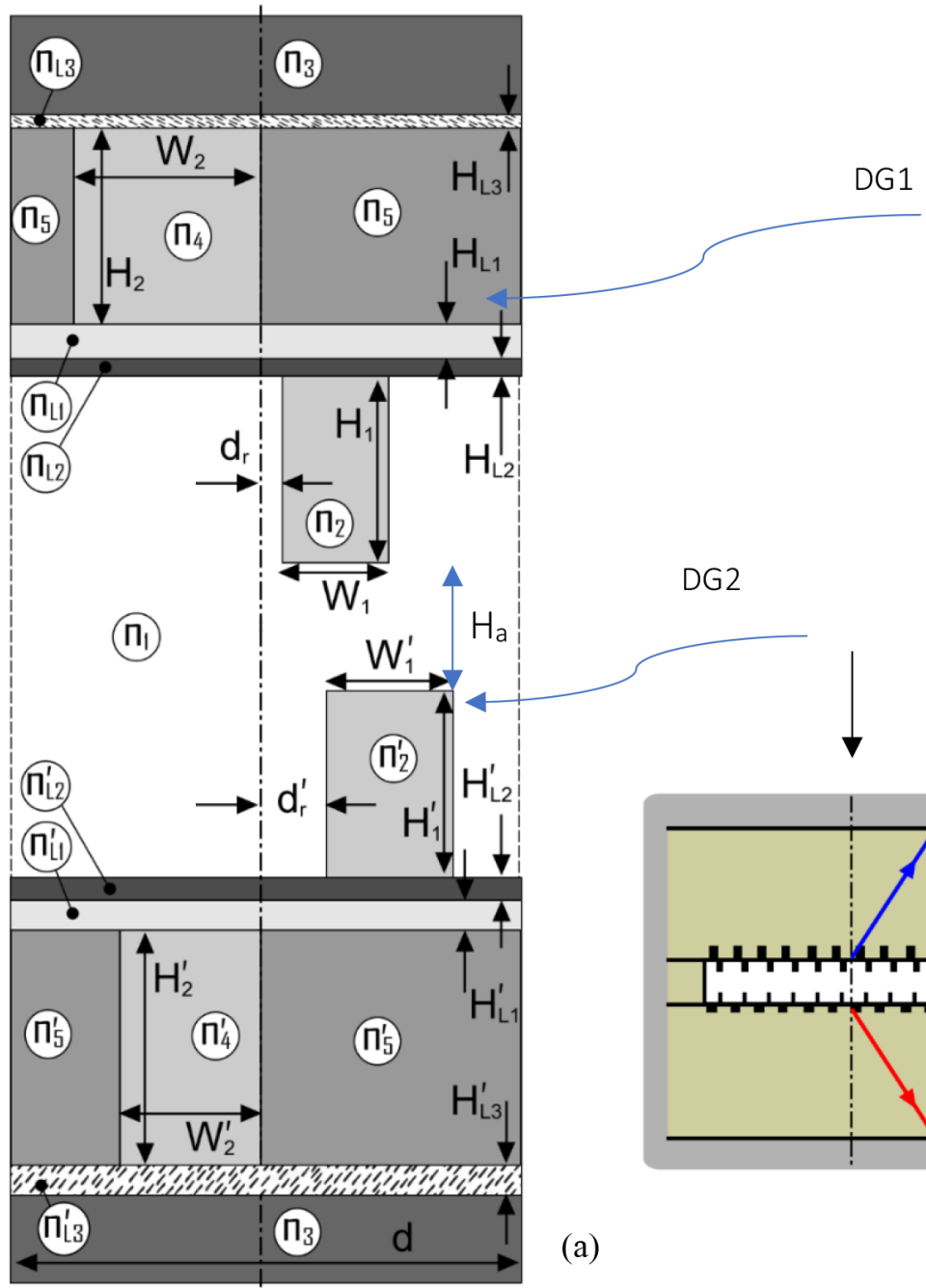

DG2

Fig. 8. (a) Cross-section views of the unit cell of the metagrating. (b) Schematic side view illustrating geometry and distribution of diffraction order propagation of an example metagrating.

wavelength and an angular range covering the portion of FoV which was not in-coupled by the first grating. Assuming that $\Phi_{1, \text { red }}^{G} \approx \Phi_{2, \text { red }}^{G} \approx 90^{\circ}$, we get

$$
d_{2} \approx \frac{M_{T} \lambda_{\text {red }}}{n_{3 R}+n_{1} \sin \left(\theta_{1, \text { red }}^{G}-\Delta \theta\right)}
$$

where $\theta_{1, \text { red }}^{G} \approx \sin ^{-1}\left(\left(n_{3 R}-\frac{M_{R} \lambda_{\text {red }}}{d_{1}}\right) / n_{1}\right), M_{T}$ corresponds to the diffraction order of the second diffraction grating in the example metagrating, $n_{3 R}$ is a refractive index of the waveguide at red color wavelength, $\Delta \theta$ is angular overlapping for the grazing incident rays in-coupling by the reflective grating and grazing incident rays in-coupling by the transmissive grating $\left(\theta_{2, \text { red }}^{G} \approx \theta_{1, \text { red }}^{G}-\Delta \theta\right)$.

It was demonstrated that to in-couple all three colors the gratings with substantially equal periods may be combined. For example, the period of the metagrating may be equal to $d=d_{1}=d_{2}$. If the period of the reflective diffraction grating is defined to in-couple diffraction order
$M_{R}$ and the period of the transmissive diffraction grating is defined to in-couple diffraction order $M_{T}$, the period of new metagrating is defined to in-couple reflected order $M_{R}{ }^{*}=$ $M_{R}$ and transmitted order $M_{T}{ }^{*}=M_{T}$.

An example topology of the unit cell of a metagrating is illustrated in Figure 8a. To get the unit cell of an example diffraction metagrating, we combine two plates with first (DG1) and second (DG2) diffraction gratings. The distance between the plates/substrates may be represented as $H_{1}+H_{1}^{\prime}+H_{\mathrm{a}}$, where $H_{\mathrm{a}}$ is the distance between the elements. It must be filled by a material with low refractive index $n_{1}\left(n_{1}<n_{3}\right)$.

The mutual positioning of the elements of the two diffraction gratings in relation to one another inside the pitch does not affect the system performance, the proposed system is not sensitive to lateral shifts. Configuring the reflective and transmissive parts of a metagrating to in-couple fist diffraction orders $\left(M_{R}=M_{T}=1\right)$ results in a metagrating for which the first reflected order $\mathrm{R}_{1}\left(M_{R}{ }^{*}=1\right)$ and 1 st transmitted order $\mathrm{T}_{1}$ $\left(M_{T}^{*}=1\right)$ will be in-coupled into the waveguide. The 


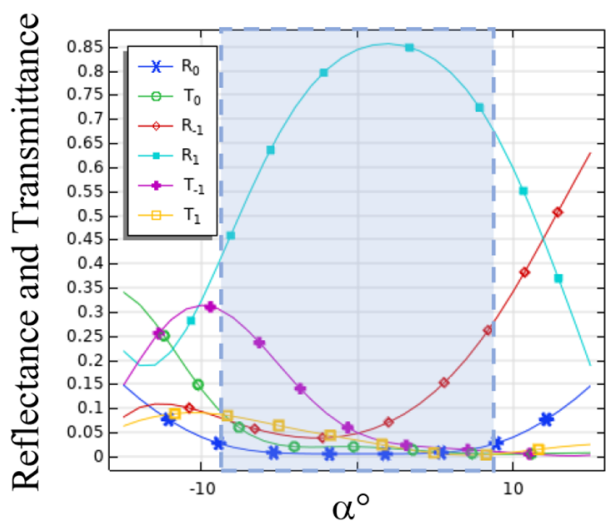

(a)

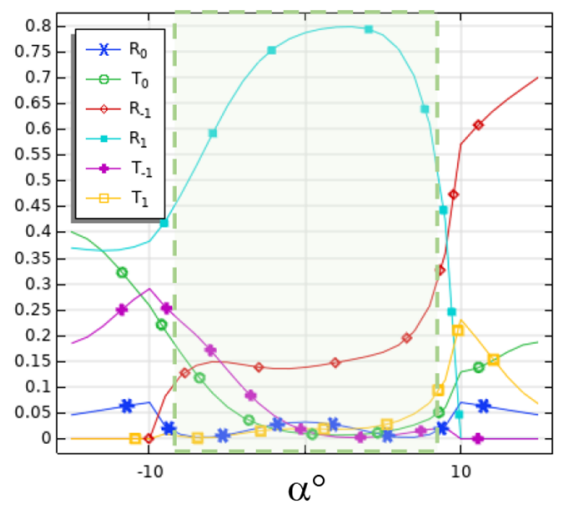

(b)

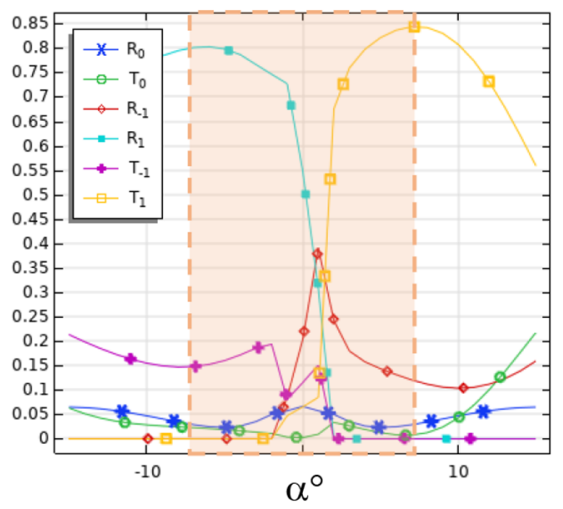

(c)

Fig. 9. Diffraction performance of the $\mathrm{TiO}_{2}$ metagrating for (a) blue, (b) green and (c) red light. The angular ranges in-coupled by the system are shaded. The incidence angles correspond to the incidence from the medium with refractive index $n_{3}$ (waveguide material).

distribution of the diffracted light inside the waveguide is illustrated schematically in Figure 8b. To prevent undesirable back reflection, it is desirable to consider the lateral size of the metagrating and the thicknesses of the waveguide.

Below we present results of numerical simulations for the metagrating with a high refractive index configured to in-couple the first reflective diffraction order and first transmissive diffraction order for TE polarization. The simulated system has been configured using $\mathrm{TiO}_{2}$ as the material of the elemets of DG1 and DG2 and sapphire $\left(\mathrm{Al}_{2} \mathrm{O}_{3}\right)$ as the material of the substrate and stop layers. For the simulated embodiment, half of the elements of DG1 and DG2 are embedded in a homogeneous dielectric host medium with a refractive index $n_{5}=n_{5}$. The host medium was $\mathrm{SiO}_{2}$ material. The presented numerical simulations take into account the dispersion of materials. Figure 9 illustrates diffraction performance of a $\mathrm{TiO}_{2}$ metagrating (the unit cell is depicted in Fig. 8a) with the following parameters: $w_{1}=w_{2}=80 \mathrm{~nm}, h_{1}=90 \mathrm{~nm}, h_{2}=80 \mathrm{~nm}, w_{1}^{\prime}=$ $w_{2}^{\prime}=120 \mathrm{~nm}, h_{1}^{\prime}=h_{2}^{\prime}=160 \mathrm{~nm}, H_{\mathrm{L} 2}^{\prime}=H_{\mathrm{L} 2}=5 \mathrm{~nm}, d_{\mathrm{r}}^{\prime}=$ $30 \mathrm{~nm}, H_{\mathrm{a}}=170 \mathrm{~nm}$. Figures $9 \mathrm{a}, 9 \mathrm{~b}$ and $9 \mathrm{c}$ shows the performance with blue $(460 \mathrm{~nm})$, green $(530 \mathrm{~nm})$ and red light $(620 \mathrm{~nm})$, respectively. The required angular ranges in-coupled by the system are shaded. We have calculated that for on-axis in-coupling $d=358.69 \mathrm{~nm}, M_{R}{ }^{*}=1$, and $M_{T}{ }^{*}=1$. The desired symmetrical $\mathrm{FoV}$ is equal to $30^{\circ}$. Some angles are overlapped to avoid missing display information for some colors [13]. Such a system achieves the desired FoV for all 3 colors using just one waveguide. For the proposed solution for blue and red colors, we can achieve a diffraction efficiency corresponding to $45-85 \%$, and an efficiency about $45-80 \%$ for green over a FoV of $30^{\circ}$. In systems in which the index of refraction of the waveguide is increased, it is possible to improve the uniformity of transmitted orders by choosing the angular ranges with more uniform distribution for each diffractive grating.

\subsection{Single-mode transmissive metasurface}

Third proposed design provides a full RGB single waveguide system based on a single-mode transmissive meta-surface. In comparison with the case described in Section 2.2, the fabrication process will avoid the bonding of 2 plates. The proposed system can be also configured to get on- and off-axis in-coupling for the desired FoV of the device. The configuration is selected to create transmissive metasurface in-couplers based on combination of transmissive diffraction gratings providing nonsymmetrical response. Constitutive parts of the example metasurface are nonsymmetrical transmissive diffraction gratings described in Section 2.2 (see Fig. 6a). The second transmissive diffraction grating is different from the first one in that it has a different pitch size, but the geometrical structure is of the same shape. The equations for the period of two transmissive diffraction gratings can be presented in such form:

$$
\begin{aligned}
& d_{1}=\frac{M_{1 T} \lambda_{\text {blue }}}{n_{3 B}+n_{1} \sin \theta_{1, \text { blue }}^{G}} \\
& d_{2} \approx \frac{M_{2 T} \lambda_{\text {red }}}{1+n_{1} \sin \left(\theta_{1, \text { red }}^{G}+\Delta \theta\right)},
\end{aligned}
$$

where $\left|\theta_{1, \text { blue }}^{G}\right|=\left|\theta_{m}+\gamma_{m}\right|, \quad \theta_{1, \text { red }}^{G} \approx \sin ^{-1}\left(\left(-n_{3 R} \sin \Phi_{1, \text { red }}^{G}+\right.\right.$ $\left.\left.\frac{M_{1 T} \lambda_{\text {red }}}{d_{1}}\right) / n_{1}\right), M_{1 T, 2 T}$ correspond to the diffraction orders of the first and second diffraction gratings of the metasurface, $\Phi_{1, \text { red }}^{G}$ is chosen approximately equal to $75^{\circ}-90^{\circ}$.

To estimate the effectiveness of the full system, a metasurface was simulated with a unit cell comprising three elements of DG1 and two elements of DG2. In the example, the period of the metasurface is equal to $d=3 d_{1}=2 d_{2}$. In a case where the period of the diffraction grating DG1 is selected to in-couple diffraction order $M_{1 T}$ and the period of transmissive diffraction grating DG2 is selected to in-couple diffraction order $M_{2 T}$, the period of the resulting metasurface is defined to in-couple transmitted order $M_{1 T}{ }^{*}=3 M_{1 T}$ and transmitted order $M_{2 T}{ }^{*}=2 M_{2 T}$. Configuring the constitutive parts of a metasurface to in-couple fist diffraction orders $\left(M_{1 T}=\right.$ $\left.M_{2 T}=1\right)$ results in a metasurface for which the third transmitted order $\mathrm{T}_{3}\left(M_{1 T^{*}}{ }^{*}=3\right)$ and second transmitted order $\mathrm{T}_{2} \quad\left(M_{2 T^{*}}{ }^{*} 2\right)$ will be in-coupled into the waveguide. 


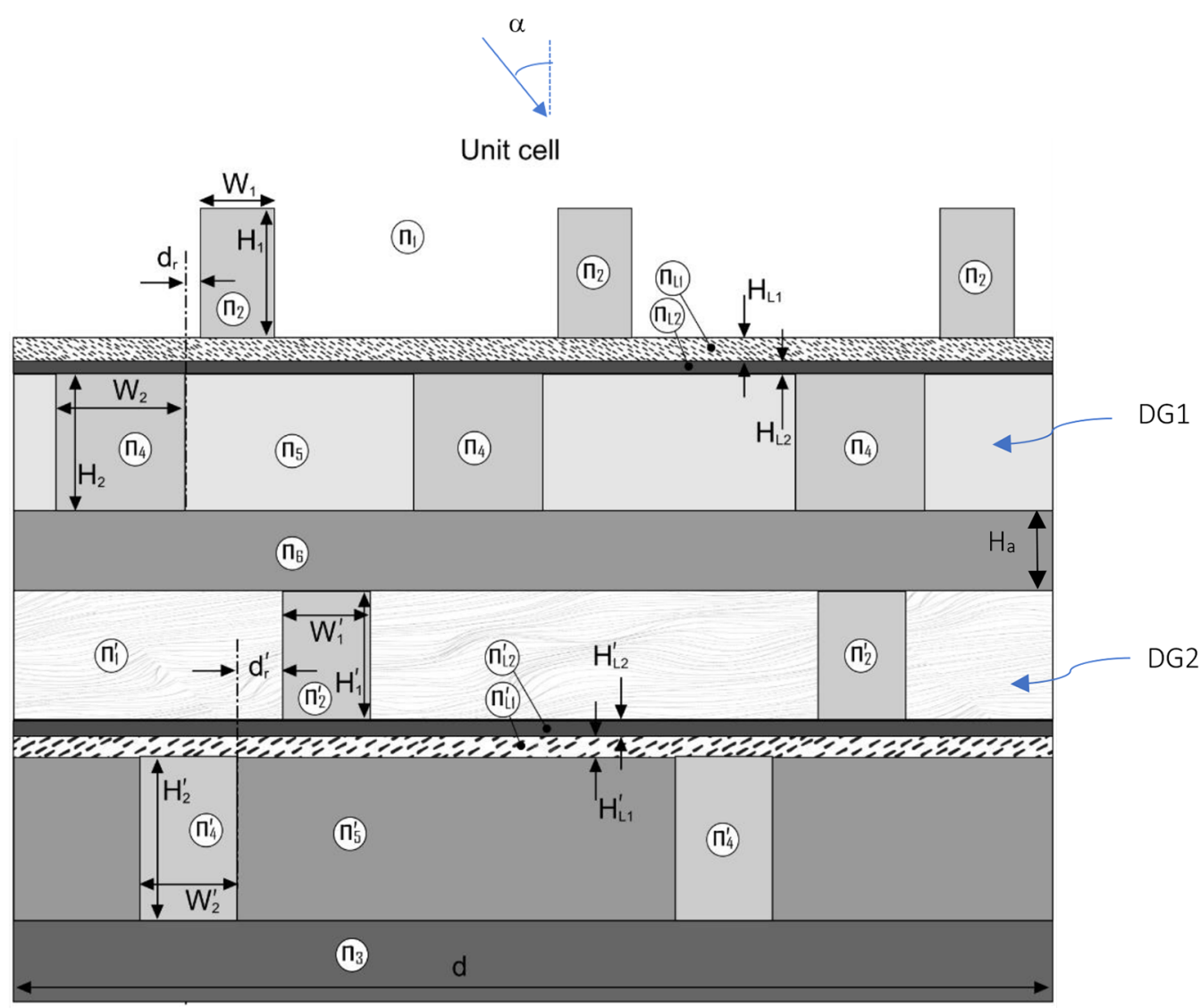

Fig. 10. Cross-sectional view of the unit cell of the metasurface on top of the waveguide.

As an example, consider a transmissive metasurface on the top of the waveguide with a unit cell presented in Figure 10. This cross-sectional view may correspond to the high refractive index $\left(n_{2}\right.$ and $\left.n_{4}\right)$ elements of the first diffraction grating (DG1) and to the high refractive index $\left(n_{2}^{\prime}\right.$ and $\left.n_{4}^{\prime}\right)$ elements of the second diffraction grating (DG2). In this example, the metasurface is placed on a homogeneous dielectric plate with a refractive index $n_{3}$ $\left(n_{2,4}\right.$ and $\left.n_{2,4}^{\prime}>n_{3}\right)$. The space between two gratings is filled up by the material with refractive index $n_{6}$. In this example, the distance between the two gratings is equal to $H_{\mathrm{a}}$. To improve the uniformity of transmitted diffraction orders and additionally increase the transmittivity of in-coupled diffraction order, we consider phase modifying layers with the thickness $H_{\mathrm{L} 2}$ and $H_{\mathrm{L} 1}$ and refractive indexes $n_{\mathrm{L} 2}$ and $n_{\mathrm{L} 1}$ placed between the high refractive index elements of the gratings. To simplify the fabrication process, we use so-called stop layers between this thin layer and top elements of the gratings. In this example, $n_{\mathrm{L} 1}$ and $n_{\mathrm{L} 2}^{\prime}$ are the stop layer material refractive indexes, and $H_{\mathrm{L} 1}$ and $H_{\mathrm{L} 2}$ are the thicknesses of these layers.

The angular distribution of the diffracted light inside the waveguide is presented in Figure 11. The schematics in Figure 11 illustrate the angular space for each color (starting from the blue color). For blue color (Fig. 11a) the coupled portion of light corresponds to the 3rd transmitted order. For green and red colors (Fig. 11b, c), the illustration shows the portion of light coupled by this waveguide corresponding to the third and second transmitted orders of the metasurface.

Below is presented a set of numerical simulations for an example transmissive metasurface with a high refractive index configured to in-couple 2nd and 3rd transmissive diffraction orders for TE polarization. Figure 12 illustrates simulated diffraction performance of the $\mathrm{TiO}_{2} / \mathrm{AlAs}$ transmissive meta-surface using the unit cell depicted in Figure 10 with the following parameters: $d=980 \mathrm{~nm}$, $w_{1}=80 \mathrm{~nm}, \quad w_{2}=100 \mathrm{~nm}, h_{1}=h_{2}=100 \mathrm{~nm}, \quad H_{\mathrm{L} 1}=5 \mathrm{~nm}$, $H_{\mathrm{L} 2}=0 \mathrm{~nm}, \quad w_{1}^{\prime}=60 \mathrm{~nm}, \quad w_{2}^{\prime}=80 \mathrm{~nm}, \quad h_{1}^{\prime}=180 \mathrm{~nm}$, $h_{2}^{\prime}=200 \mathrm{~nm}, \quad H_{\mathrm{L} 1}^{\prime}=0 \mathrm{~nm}, \quad H_{\mathrm{L} 2}^{\prime}=0 \mathrm{~nm}, \quad d_{\mathrm{r}}=-30 \mathrm{~nm}$, $d_{\mathrm{r}}^{\prime}=10 \mathrm{~nm}, H_{\mathrm{a}}=110 \mathrm{~nm}$. Sapphire is the material of the waveguide. The FoV extends between $-15^{\circ}$ and $+15^{\circ}$ for a total $\mathrm{FoV}$ of $30^{\circ}$. For the simulation, $n_{1}=1$ (air), $n_{2}=n_{4}$ and this refractive index corresponds to $\mathrm{TiO}_{2}, n_{2}^{\prime}=n_{4}^{\prime}$ and this refractive index corresponds to AlAs, $n_{5}=n_{1}{ }_{1}$ and it is $\mathrm{SiO}_{2}$ material, $n_{3}=n_{\mathrm{L} 1}=n_{5}^{\prime}=n_{6}$ and it is the material of a waveguide $\left(\mathrm{Al}_{2} \mathrm{O}_{3}\right)$.

It was obtained that for the blue color we can get about $25-65 \%$ of an average diffracted efficiency, for the green color we can achieve a diffraction efficiency corresponding to $20-45 \%$, and of about $30-75 \%$ for the red. 


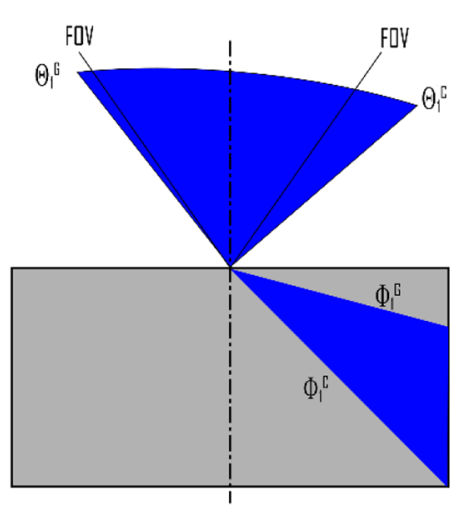

(a)

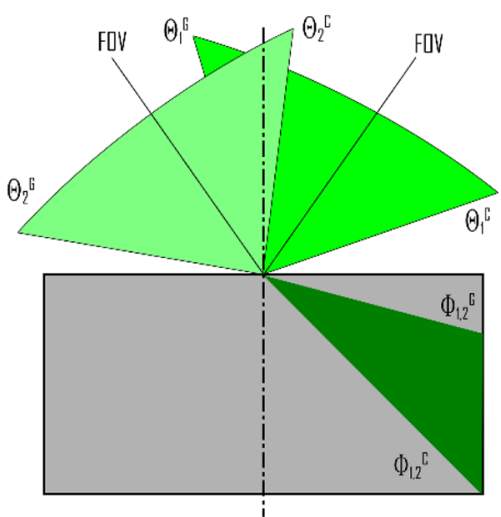

(b)

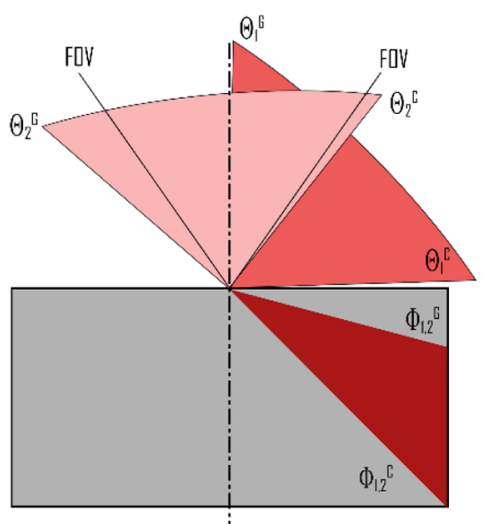

(c)

Fig. 11. Schematics of a single waveguide system with metasurface solution illustrating angles of incident and diffracted light for transmissive diffraction gratings for three different colors: (a) blue, (b) green, (c) red.

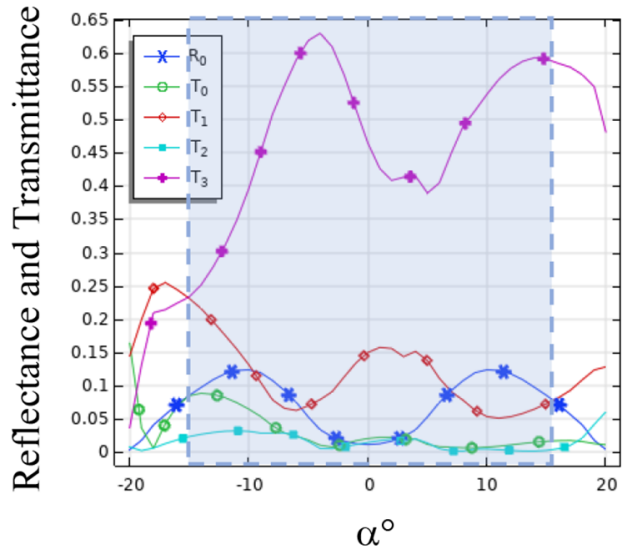

(a)

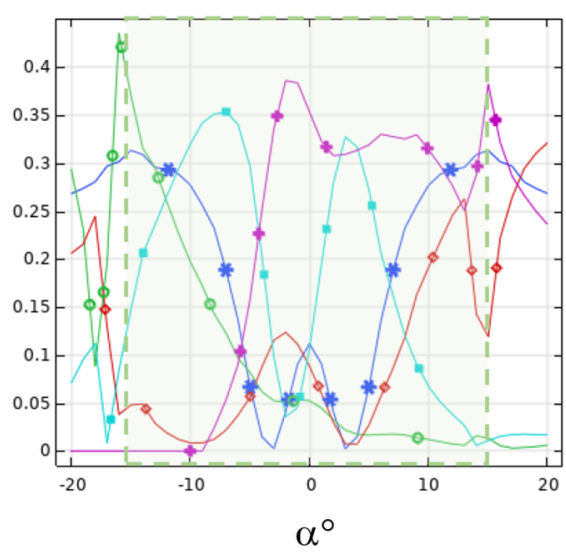

(b)

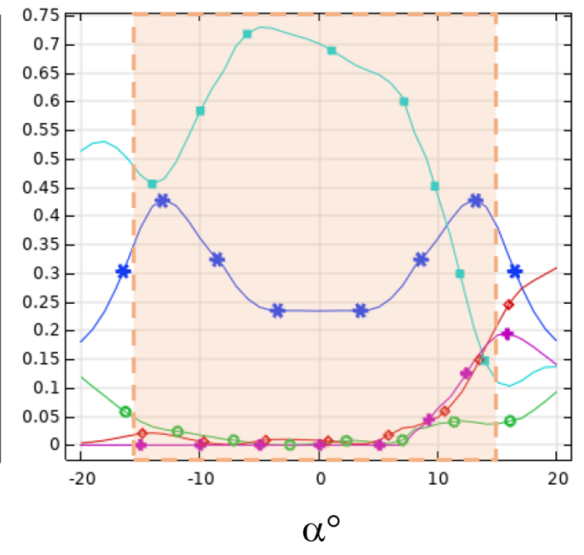

(c)

Fig. 12. Diffraction performance of the $\mathrm{TiO}_{2} / \mathrm{AlAs}$ transmissive metasurface using the unit cell depicted in Figure 10 for an example set of parameters for (a) blue, (b) green and (c) red light.

\section{Full color eye-pupil-expander}

In this section we describe a new full-color EPE component which is compatible with dual-mode metagrating in-coupler.

In Figure 13a we schematically present the full system architecture based on dual-mode metagrating elements embedded inside the waveguide. We assume that each metagrating element represents a combination of two diffraction gratings oriented at some specific angle. We define that both in-coupling gratings have the same orientation $\left(\Phi_{i n c}=90^{\circ}\right)$. They diffract the positive order toward the left-hand side of the figure and the negative order toward the right-hand side. Then we have two similar EPE metagratings oriented symmetrically w.r.t. the $y$-axis. Each EPE metagrating includes two diffraction gratings which can have different orientation. The EPE metagratings diffract zero orders to expand the pupil horizontally, and non-zero order to deviate the image along the y-axis. For this architecture the out-coupling grating also expands the pupil in the vertical direction.
Let us calculate the pitches of the EPE and outcoupling gratings for one waveguide full color system. To determine the desired grating periods of the EPE and outcoupling gratings, we start with the basic equation for a diffraction grating in conical mounting. The well-known grating equations for the EPE diffraction gratings can be written as:

$$
\begin{aligned}
& n_{2} \sin \theta_{e} \cos \varphi_{e}+n_{2} \sin \theta_{d} \cos \varphi_{d}=\frac{N \lambda}{d_{e p e}} \sin \Phi_{\text {epe }} \\
& n_{2} \sin \theta_{e} \sin \varphi_{e}+n_{2} \sin \theta_{d} \sin \varphi_{d}=\frac{N \lambda}{d_{e p e}} \cos \Phi_{\text {epe }}
\end{aligned}
$$

Here, $\theta_{d}$ is the incident polar angle diffracted by the incoupling grating; $\theta_{e}$ is the polar angle diffracted by the EPE grating for diffraction order $N$ of EPE grating; $\varphi_{d}$ and $\varphi_{e}$ are incident (angle diffracted by the in-coupling grating) and diffracted azimuth angles, $d_{e p e}$ is the period of EPE diffraction grating, $\Phi_{\text {epe }}$ is the EPE diffraction grating orientation angle. Let us remind that, the deviating EPE grating will limit the 

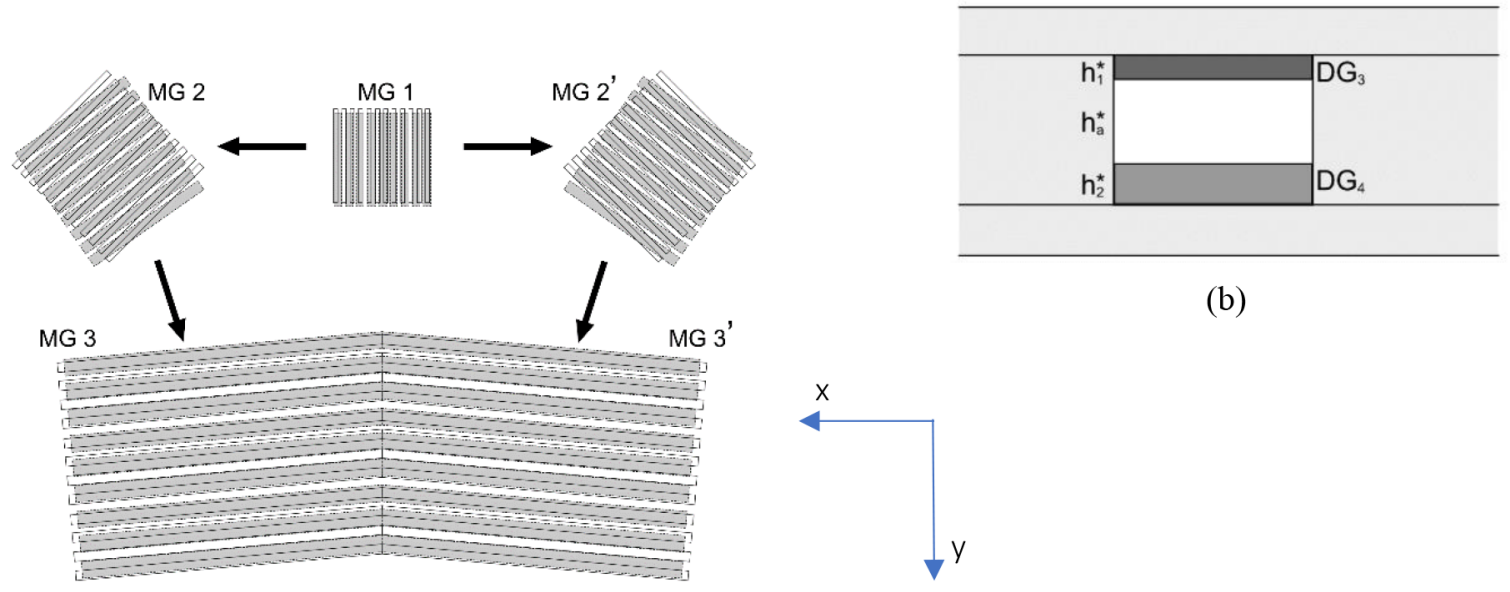

(b)

(a)

Fig. 13. (a) Perspective view of the full color waveguide having dual-mode metagrating in-coupler (MG1), EPE system (MG2 and MG2') and out-coupling grating system (MG3 and MG3'); (b) Side view of a waveguide showing internal EPE metagrating MG2.

vertical FoV of the total system [20]. This limitation is connected with the fact, that the diffracted angles $\theta_{e}$ must be also kept above the TIR limit angle. This fact must be precisely analyzed for the full color system solution.

The out-coupling system is described by such system of diffraction grating equations:

$$
\begin{aligned}
& n_{1} \sin \theta_{o} \cos \varphi_{o}+n_{2} \sin \theta_{e} \cos \varphi_{e}=\frac{L \lambda}{d_{\text {out }}} \sin \Phi_{\text {out }} \\
& n_{1} \sin \theta_{o} \sin \varphi_{o}+n_{2} \sin \theta_{e} \sin \varphi_{e}=\frac{L \lambda}{d_{\text {out }}} \cos \Phi_{\text {out }},
\end{aligned}
$$

where $\theta_{o}$ is the polar angle diffracted by the out-coupling grating for diffraction order $L$ of this grating; $\varphi_{o}$ is diffracted azimuth angle, $d_{\text {out }}$ is the period of out-coupling diffraction grating, $\Phi_{\text {out }}$ is the grating orientation angle.

Based on the general analysis of distortion free condition for the grating vectors of the system and assuming that $\Phi_{i n c}=90^{\circ}$ we get

$$
\begin{aligned}
\frac{d_{\text {out }}}{L} & =\frac{d_{\text {inc }}}{M} \cos \Phi_{\text {out }}\left(\tan \Phi_{\text {out }}-\tan \Phi_{\text {epe }}\right), \\
\frac{d_{\text {epe }}}{N} & =\frac{d_{\text {inc }}}{M} \cos \Phi_{\text {epe }}\left(\tan \Phi_{\text {out }}-\tan \Phi_{\text {epe }}\right) .
\end{aligned}
$$

Equations (10) are the formulas connecting pitches of EPE and out-coupler with the period of in-coupler and parameters of full waveguide system.

Our solution corresponds to the combination of $2 \mathrm{EPE}$ gratings DG3 and DG4 embedded into the waveguide. Such combination of EPE diffractive gratings can be considered as EPE metagrating solution MG2 (see Fig. 13a for full system design). To get the pitches of the constitutive parts of such EPE metagrating $\left(d_{\text {epe1,epe } 2}\right)$ we can also use equation (9) assuming that the pitches of in-coupling system $d_{\text {inc1,inc2 }}$ are calculated using the equations (2) and (3). To avoid undesirable mutual effect of the grating combination we must properly optimize the distance between the elements of these gratings. For the optimizations proposed below we assume that this distance could be above one wavelength.

To get the EPE metagrating MG2 we combine two plates with first EPE (DG3) and second EPE (DG4) diffraction gratings. We assume that the distance between the plates/substrates is equal to $h^{*}{ }_{1}+h^{*}{ }_{2}+h^{*}{ }_{\mathrm{a}}$, where $h^{*}$ a is the distance between the elements, and $h^{*}{ }_{1}$ and $h^{*}{ }_{2}$ are the heights of the gratings (see Fig. 13b). This distance between the elements of two gratings and substrates is fulfilled by the material with low refractive index $n_{1}$.

Table 1 shows the parameters of the full system including the periods of out-coupling gratings DG7 and DG8. We assume that the EPE gratings are oriented differently. Orientation of the EPE gratings DG3 and DG4 were optimized to get maximal vertical FoV of the full system.

Analyzing the parameters of this full system, we demonstrate that for such a system horizontal FoV is equal to $\Delta Q^{\mathrm{H}}=74.6^{\circ}$. Analyzing the system of grating equations, we get that for blue and red color vertical $\mathrm{FoV}$ is equal to $\Delta Q^{V}=40^{\circ}$. Taking into account that at green color wavelength full angular range will be split between the gratings DG1 and DG2, we must check the out-coupled angular bandpasses at green color. Numerical analysis of the possible horizontal and vertical FoVs for this system of metagratings is presented in Figure 14. In this figure the angular ranges kept above the TIR limit angle and below the grazing limit for corresponding polar angles before and after EPE are marked by yellow. Due to the EPE gratings' limitation of vertical FoV at green color wavelength the full vertical FoV of the system will be decreased to $\Delta Q^{V}=24^{\circ}$.

To duplicate a single pupil into many, each EPE grating in MG2 and MG2' combination represents a combination of multiple subgratings with the same pitch but different diffraction efficiency changing gradually from minimal value to maximal (see Fig. 15a). The general topology of the unit cell of symmetrical reflective 

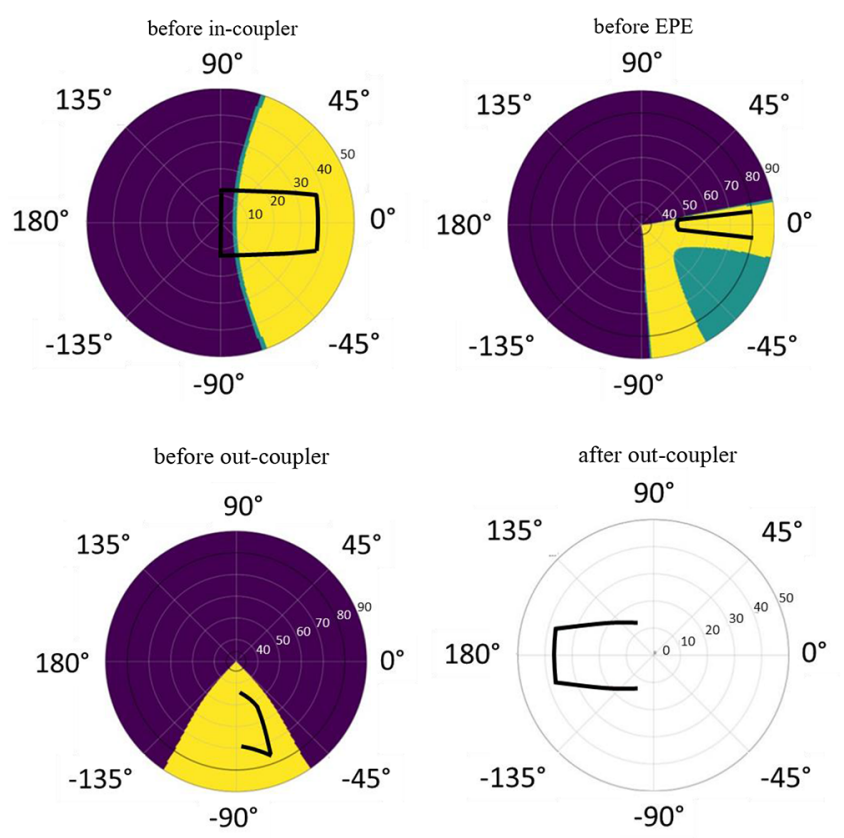

(a)
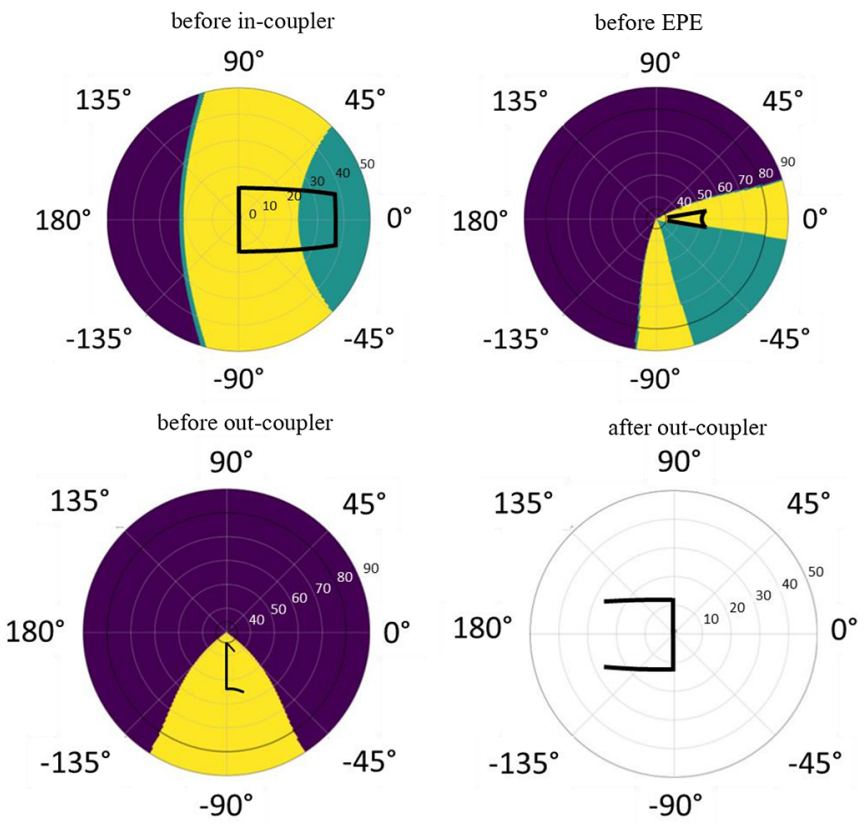

(b)

Fig. 14. Possible FoV for the system of metagratings. Parameters of the diffraction gratings are presented in Table 1. Simulations corresponds to the systems (a) DG1+DG3+DG7 and (b) DG2+DG4+DG8 at green color wavelength. In the pictures (a)-(b) top left: Angular bandpass before in-coupler; top right: Angular bandpass before EPE; bottom left: Angular bandpass before out-coupler; bottom right: Angular bandpass after outcoupler; yellow band: Proper angular bandpass after diffraction; green band: Nonproper angular bandpass after diffraction; purple band: Bandpass impossible for diffraction. diffraction subgrating is illustrated in Figure 15b. This cross-section view may correspond to a high refractive index $\left(n_{3}\right)$ element on the bottom of a homogeneous dielectric media with a refractive index $n_{2}\left(n_{3}>n_{2}\right)$. Here we assume that all EPE gratings have the same structure and materials. But in general case the structure and high index material for the gratings can be different. The high refractive index element is covered by the material with lower refractive index $n_{4}\left(n_{3}>n_{4}\right)$. The full system is hosted by the homogeneous host medium with refractive index $n_{1}$. It is assumed that $n_{1}<n_{2}$ and $n_{1}<n_{4}$. $W$ and $H$ are the width and height of the high refractive index element (in general case the parameters of the high index elements for DG3 and DG4 are different). $H_{1}$ is the thickness of the layer with refractive index $n_{5}$.

\section{Conclusion}

New types of full color single waveguide solutions are presented in this work. Applying our new grating combination solutions for single- and dual-mode in-couplers, we can modify the total in-coupled FoV of the system. For the case of limited FoV of the device, we can use single-mode grating simplifying the system configuration. We have demonstrated that single-mode embedded metagrating solution and single-mode transmissive metasurface provides high diffraction uniformity and efficiency of in-coupled light for horizontal $\mathrm{FoV}$ of about $30^{\circ}$ for three RGB colors and a wafer with a refractive index of 1.76. Our full system can be optimized to get on- and offaxis in-coupling for the required FoV of the device. To achieve high FoV, we use the pupil tiling property of the grating. To develop a waveguide display system with a wide FoV, we can combine two single-mode metagratings optimized for off-axis in-coupling or we can use dual-mode metagrating solution. It was demonstrated that for the waveguide material with refractive index $1.76\left(\mathrm{Al}_{2} \mathrm{O}_{3}\right)$, we can get an overall horizontal $\mathrm{FoV}$ of $89^{\circ}$ for three colors.

A new type of full-color EPE metagrating component which is compatible with dual-mode metagrating in-coupler is analyzed. It is demonstrated that by orienting EPE gratings differently we can increase the vertical $\mathrm{FoV}$ of the full system getting an overall vertical $\mathrm{FoV}$ of $24^{\circ}$ for three colors.

Applying our new solutions, we can provide good gathering of diffracted rays for the different colors. These new metagratings are also protected from mechanical damage and degradation.

In collaboration with the French company Vmicro (Lille, France), we have started the analysis of the fabrication steps of a prototype of a dual-mode embedded metagrating solution. At the moment we have fabricated by e-beam lithography a Si-based transmissive diffractive grating which can be used as the transmissive part (grating DG2 in Fig. 4a) of the in-coupling metagrating solution. For the system design, we selected $\mathrm{Al}_{2} \mathrm{O}_{3}$ as the material of 

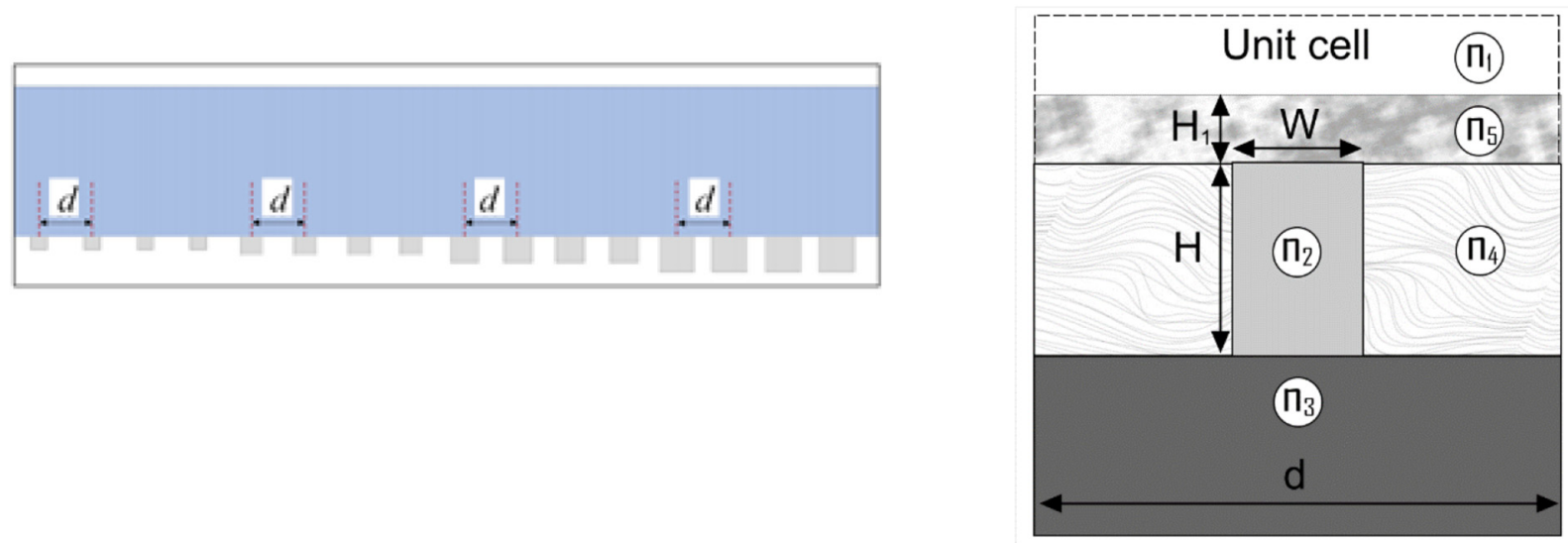

Fig. 15. (a) Single EPE grating including several subgratings; (b) cross-sectional view of reflective subgrating unit cell.

Table 1. Parameters of the full-color system of metagratings.

\begin{tabular}{lll}
\hline & Red color & Blue color \\
\hline$\lambda$ & $638 \mathrm{~nm}$ & $460 \mathrm{~nm}$ \\
$d_{\text {inc }}$ & $d_{\text {inc1 }}=382 \mathrm{~nm}$ & $d_{\text {inc } 2}=286 \mathrm{~nm}$ \\
$\Phi_{\text {inc }}$ & $\Phi_{\text {inc1 }}=90^{\circ}$ & $\Phi_{\text {inc } 2}=90^{\circ}$ \\
$d_{\text {epe }}$ & $d_{\text {epe } 1}=287 \mathrm{~nm}$ & $d_{\text {epe } 2}=226 \mathrm{~nm}$ \\
$\Phi_{\text {epe }}$ & $\Phi_{\text {epe } 1}=48.7^{\circ}$ & $\Phi_{\text {epe } 2}=52^{\circ}$ \\
$d_{\text {out }}$ & $d_{\text {out } 1}=435 \mathrm{~nm}$ & $d_{\text {out } 2}=367 \mathrm{~nm}$ \\
$\Phi_{\text {out }}$ & $\Phi_{\text {out } 1}=0^{\circ}$ & $\Phi_{\text {out } 2}=0^{\circ}$ \\
\hline
\end{tabular}

substrate. To control the etching depth of $\mathrm{Si}$, we used an additional thin $(10 \mathrm{~nm})$ layer of $\mathrm{TiO}_{2}$ placed on the top of substrate. We must take into account that the fabrication process will be affected by the exact shape and size of the plates placed between the constitutive parts of the waveguide with the gratings DG1 and DG2. We also remind that our analysis demonstrated that the mutual positioning of the elements of these 2 gratings in relation to one another inside the unit cell does not affect the system performance. This fact will also simplify the fabrication.

\section{References}

1. B.C. Kress, I. Chatterjee, Nanophotonics 10, 41 (2021)

2. T. Zhan et al., iScience 23, 101397 (2020)

3. K. Yin et al., J. Phys. Photonics 3, 022010 (2021)

4. J.-Y. Hong et al., Sci. Rep. 7, 2753 (2017)
5. J.-H. Park, S.-B. Kim, Opt. Express 26, 27076 (2018)

6. G. Li et al., Opt. Lett. 41, 2486 (2016)

7. G.-Y. Lee et al., Nat. Commun. 9, 4562 (2018)

8. M.L. Brongersma, Proc. SPIE 11080, 110800R (2019)

9. Z. Shi, W.T. Chen, F. Capasso, Proc. SPIE 10676, 1067615 (2018)

10. J. Yang et al., Opt. Lett. 41, 5426 (2016)

11. D. Cheng et al., Opt. Express 22, 20705 (2014)

12. T. Levola, P. Laakkonen, Opt. Express 15, 2067 (2007)

13. V. Drazic et al., Proc. SPIE 11765, 11765A (2021)

14. H. Mukawa et al., J. Soc. Inf. Disp. 17, 185 (2009)

15. C. Chang et al., Optica 7, 1563 (2020)

16. O. Shramkova et al., Proc. SPIE 11802, 118020D (2021)

17. M.-A. Mattelin, Opt. Express 8, 11175 (2020)

18. B. Bai et al., Appl. Opt. 49, 5454 (2010)

19. C.P. Chen et al., Displays 67, 101998 (2021)

20. V. Drazic, L. Blonde, O. Shramkova, Patent application WO2021 14 4452A1

21. Yu. Liu et al., Proc. SPIE 9449, 944914 (2014)

Cite this article as: Oksana Shramkova, Valter Drazic, Guillaume Bourcin, Bobin Varghese, Laurent Blondé, Valerie Allié, Metagrating solutions for full color single-plate waveguide combiner, EPJ Appl. Metamat. 9, 5 (2022) 